\author{
Girolamo Di Trapani \\ Daniele Mei \\ Catello Vollono \\ Alessandro Capuano \\ Diana Ferraro
}

\section{Antiepileptic drugs in the treatment of headache: neuroprotective effect or something else?}

G. Di Trapani $(\bowtie) \cdot$ D. Mei • C. Vollono

A. Capuano $\cdot$ D. Ferraro

Headache Center, Institute of Neurology, Policlinico 'A. Gemelli',

Largo A. Gemelli 8, I-00168 Rome, Italy e-mail: girolamo.ditrapani@rm.unicatt.it

Tel.: +39-06-30154435

Fax: +39-06-35501909

\begin{abstract}
The hypothesis that cortical hyperexcitability may play an important role in the physiopathology of migraine has lead to the therapeutic use of antiepileptic drugs in headache prophylaxis. Cortical hyperexcitability is due to an imbalance between neuronal inhibition, mediated by gamma-aminobutyrric acid (GABA), and neuronal excitement, mediated by excitatory aminoacids. It becomes therefore clear how cortical excitability may be modulated by acting on mechanisms such as the synthesis and metabolism of GABA and on targets such as GABA and glutamate receptors and on sodium and calcium channels. The question is, whether, thanks to their tolerability and rapidity of action, these should be considered as first-choice prophylaxis
\end{abstract}

drugs rather than simply as alternative drugs. Thanks to their action on the key-mechanisms implicated in the genesis and maintenance of pain, valproic acid, gabapentin, topiramate, levetiracetam and lamotrigine have all the requisites in terms of efficacy, tolerability and rapidity of action that are requested from a drug in order to be considered a firstchoice drug rather than simply an alternative to the drugs currently used. The expansion, however, of medical areas in which antiepileptic drugs are prescribed and the growing number of patients using them, require an increased sensitivization in order to avoid their incorrect use.

Key words Antiepileptic drugs • Migraine prophylaxis $\cdot$ Cortical hyperexcitability

\section{Introduction}

In recent years, new-generation antiepileptic drugs have found a place among classic headache prophylaxis drugs prescribed by the guidelines of the International Headache Society, such as beta-blockers, calcium antagonists and tricyclic antidepressants.
The question is whether, thanks to their tolerability and rapid onset of action, these drugs should be considered as first-choice prophylaxis medication rather than simply as alternative drugs.

The hypothesis that cortical hyperexcitability may play an important role in the physiopathology of migraine has led to the therapeutic use of antiepileptic drugs [1-3]. 
Cortical hyperexcitability is caused by an imbalance between neuronal inhibition, mediated by gammaaminobutyric acid (GABA), and neuronal excitement, mediated by excitatory amino acids.

It therefore becomes clear how cortical excitability may be modulated by acting on mechanisms such as the synthesis and metabolism of GABA and on targets such as GABA and glutamate receptors as well as on sodium and calcium channels.

An abnormal neuronal excitability may trigger a depolarization, considered to be responsible for the 'spreading depression' [1] and for the abnormal entry of calcium that can cause a mitochondrial accumulation of calcium.

Thus, ion channels seem to play a very specific role. In particular voltage-dependant ion channels, are important for neuronal function because they modulate neuronal excitability [4]. The interaction between regulatory proteins, neurotransmitters such as serotonin, and other receptors plays a role in the constant regulation of the $\mathrm{P} / \mathrm{Q}$ calcium channel function.

Ion channels, in particular the $\mathrm{L}$ sub-group of voltagedependant channels, are important for the genesis of calcium-mediated action potentials. Their function includes the control and maintenance of the electric potentials across cell membranes, signal transductions and the regulation of action potentials in the dendrites as they permit the entry of calcium in the cell and, thus, the release of neurotransmitters [5].

The modes of action of antiepileptic drugs in headache can be summarized as functioning via two mechanisms. A given antiepileptic drug may act by only one or by both mechanisms:

- The suppression of neuronal hyperexcitability that, in turn, inhibits spreading depression.

- The modification of the dysfunction of voltage-dependant ion channels (consisting in the alteration of the inactivation gating system of sodium, potassium and calcium channels that generates an instability in neuronal excitability).

We can therefore identify some 'key targets' on which antiepileptic drugs may act determining a positive effect in the control of a headache attack:

a. The possibility of modulating excitatory pathways mediated by glutamate receptors. b. The possibility of inhibiting repeated firing of structures implicated in the processing of head pain (kindling phenomenon).

\section{Which antiepileptic drugs are currently used and what is their mode of action?}

During the past 7 years, nearly all drugs used for epilepsy have been experimented on using different scientific methods, often only in 'open-label' studies.

The only antiepileptic drugs that have proven to be truly efficacious in the prophylactic treatment of primary headaches are: valproic acid, gabapentin, topiramate, levetiracetam and lamotrigine. The efficacy of these drugs has been proven, but with varying clinical validity, as shown in Table 1.

If we closely inspect the modes of action of single antiepileptic drugs, we can delineate their neuroprotective effect and, for some of them, their adjunctive properties, considering the rational basis for their use and their key targets.

\section{Modes of action of antiepileptic drugs in headache and their neuroprotective effect}

Valproic acid [5, 7-10]

1. Inhibition of GABA-aminotransferase, enzyme implicated in the degradation of GABA.

2. Increased synthesis of GABA, through the modulation of the glutamic-acid decarboxylase, and decrease in glutamate concentrations.

3. Suppression of the depolarization mediated by the activation of NMDA-glutamate receptors.

4. Activation of GABA receptors in the dorsal raphe nucleus and subsequent reduced firing from serotoninergic neurons.

5. Inactivation of voltage-dependant sodium channels.

6. Inhibition of GABA re-uptake.

7. Blockage of $\mathrm{T}$ calcium channels.

Table 1 Efficacy of antiepileptic drugs in the treatment of primary headaches

\begin{tabular}{lllll}
\hline & Evidence level & Scientific validity & Clinical validity & Clinical features of headache \\
\hline Gabapentin & A & ++ & ++ & Migraine without aura \\
Sodium valproate & A & +++ & ++ & Migraine with aura \\
Topiramate & B & ++ & ++ & Migraine with and without aura \\
Lamotrigine & B & ++ & ++ & SUNCT syndrome \\
Levetiracetam & B & + & + & Migraine without aura \\
\hline
\end{tabular}


Furthermore, the following mechanisms of action have been discovered:

- Modulation of trigeminal nociceptive neurons in the meninges.

- Blockage of sterile neurogenic inflammation, through a $\mathrm{GABA}_{\mathrm{A}}$ receptor-mediated mechanism, with a reduction of substance $\mathrm{P}$ and plasma protein dural extravasation.

\section{Gabapentin [5, 11, 12]}

1. Reduction of neuronal hyperexcitability through the increase of GABA synthesis and its non-vesicular release.

2. Modulation of voltage-dependant ion channel dysfunction through a high-affinity binding to the $\alpha 2 \delta$ voltage-dependant calcium channel site which regulates the increase of the calcium ion flux.

3. Reduction of glutamate concentration through the inhibition of aminotransferase and the stimulation of glutamic dehydrogenase.

4. Increase of serum serotonin levels.

Topiramate $[5,13,14]$

1. Reduction of neuronal hyperexcitability through a GABAergic potentiation mediated by GABA-dependant chlorine flux.

2. Modulation of the voltage-dependant ion channel dysfunction through the blockage of sodium and calcium voltage-dependant ion channels.

3. Antagonism of AMPA/kainate-type glutamate receptors.

\section{Lamotrigine [15]}

1. Blockage of sodium voltage-dependant ion channels.

2. Stabilization of neuronal membranes through the inhibition of the release of glutamate.
Levetiracetam $[16,17]$

1. Inhibition of $\mathrm{L}$ and $\mathrm{N}$ calcium voltage-dependant ion channels.

2. Action on perimeningeal vasodilation and on neurogenic inflammation.

The dosage of antiepileptic drugs in the treatment of headache is around $50 \%$ of the dose used in epilepsy, since clinical evaluations have demonstrated that there is no correlation between increasing dosages and clinical efficacy.

The mean dosage of each antiepileptic drug is the following: valproic acid, 600-1,200 mg/day; gabapentin, 900-1,200 mg/day; topiramate, $100 \mathrm{mg} /$ day; levetiracetam, 1,500 mg/day; and lamotrigine, $100 \mathrm{mg} /$ day.

\section{Conclusions}

On the basis of their action on key-mechanisms, valproic acid, gabapentin, topiramate, levetiracetam and lamotrigine have all the requisites in terms of efficacy, tolerability and rapid onset of action to be considered first-choice drugs rather than simply an alternative to the drugs currently used.

However, with the current availability of antiepileptic drugs for the prophylaxis of headache, particular attention should be paid to the correct indications for their use and also to their side-effects that can invalidate therapeutic results.

Although these adverse effects are often correlated to the dosage, important side effects may also occur at the dosages recommended for headache prophylaxis.

Furthermore, the widening of medical areas in which antiepileptic drugs are prescribed and the growing number of patients using them requires an increased sensitization in order to avoid their incorrect use.

Lastly, it should be underlined that the non-homogeneous therapeutic schemes found in the literature suggest the need of adopting algorithms so as to avoid empiric treatments, in order to permit comparisons and to confirm the efficacy of therapeutic regimes that are suggested by preliminary studies. Their manageability and rapid onset of action should also be verified.

\section{References}

1. Welch KMA, D'Andrea G, Tepley N (1990) The concept of migraine as a state of central neuronal hyperexcitability. Neurol Clin 8: 817-828
2. Aurora SK, Ahmad BK, Welch KMA (1998) Transcranial magnetic stimulation confirms hyperexcitability of occipital cortex in migraine. Neurology 50:1111-1114
3. Aurora SK, Al-Sayeed F, Welch KMA (1999) The cortical silent period is shortened in migraine with aura. Cephalalgia 19:708-712 
4. Tepper SJ, Rapoport A, Sheftell F (2001) The pathophysiology of migraine. Neurology 7(5):279-286

5. Cutrer FM (2001) Antiepileptic drugs: how they work in headache. Headache 41[Suppl 1]:3-11

6. Post RM, Silberstein SD (1994) Shared mechanisms in affective illness, epilepsy and migraine. Neurology 44:S37-S47

7. Löesher W (1999) Valproate: a reappraisal of its pharmacodinamic properties and mechanisms of action. Prog Neurobiol 41:31-59

8. Cutrer FM, Limmroth V, Moskowitz MA (1997) Possible mechanisms of valproate in migraine prophylaxis. Cephalalgia 41:93-100
9. Cutrer FM, Limmroth V, Ayata C, Moskowitz MA (1995) Attenuation by valproate of c-fos immunoreactivity in trigeminal nucleus caudalis induced by intracisternal capsaicin. Br J Pharmacol 116:3199-3204

10. Lee WS, Limmroth V, Ayata $C$ et al (1995) Pripheral GABA $A_{a}$ receptormediatd effects of sodium valpoate on dural plasma protein extravasation to substance $\mathrm{P}$ and trigeminal stimulation. Br J Pharmacol 116:1661-1667

11. Taylor CP (1998) Mechanism of action of Gabapentin. Drugs today 34[Suppl D]:3-11

12. White HS (1999) Comparative anticonvulsant and mechaniscistic profile of the estabilished and newer antiepileptic drugs. Epilepsia 41[Suppl 5]:S2-S10
13. White HS, Brown SD, Wolf HH et al (1994) The anticonvulsant topiramate potentiates GABA-evoked chloride current in mouse cortical neurons. Epilepsia 35[Suppl 8]:67

14. Jensen TS (2002) Antionvulsants in neuropathic pain: rationale and clinical evidence. Eur J Pain 6[Suppl A]:61-68

15. McNamara (1996) Drugs acting on the central nervous system. In: Harman G, Limbird LE, Morinoff PB, Ruddon RW (eds) Goodman and Gilman's pharmacological basis of therapeuthics, 9th edn. Mc Graw-Hill, New York, pp 461-486

16. Radtke RA (2001) Pharmacokunetics of levetiracetam. Epilepsia 42[Suppl 4]:24-27

17. Nash EM, Sangha KS (2001) Levetiracetam. Am J Health Pharm 58(13):1195-1199 